\title{
INFLUÊNCIA DO METILMERCÚRIO NO CRESCIMENTO E DESENVOLVIMENTO FETAL
}

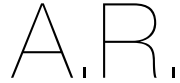
ARTIGO DE REVISÃO

1 Licenciatura em Dietética e Nutrição, Escola Superio de Tecnologia da Saúde de Coimbra, Instituto Politécnico de Coimbra, Rua 5 de Outubr Martinho do Bispo, Apartado 7006, 3040-854 Coimbra, Portugal

2 Unidade CientíficoPedagógica de Dietética e Nutrição, Escola Superior de Tecnologia da Saúde de Coimbra, Instituto Politécnico de Coimbra, Rua 5 de Outubro - São Martinho do Bispo, Apartado 7006, 3040-854 Coimbra Portugal

*Endereço para correspondência:

Carla Mustra

Rua 5 de Outubro - São Martinho do Bispo, Apartado 7006 , 3040-854 Coimbra, Portugal cmustra@gmail.com

Histórico do artigo:

Recebido a 15 de junho de 2021 Aceite a 1 de setembro de 2021

\author{
INFLUENCE OF METHYLMERCURY ON FETAL GROWTH AND \\ DEVELOPMENT
}

Carla Mustra ${ }^{1 *}$; Jessica Rodrigues'; Natale Teixeira'; Sílvia Gariso'; Ana Baltazar

RESUMO

A gravidez é um período muito sensível à exposição ambiental de metais pesados, nomeadamente ao metilmercúrio, uma vez que este é capaz de atravessar a barreira placentária e hematoencefálica, prejudicando o crescimento e desenvolvimento fetal. Com esta revisão pretende-se compreender o metabolismo do mercúrio e o seu impacto na saúde das gestantes e no desenvolvimento fetal e identificar os alimentos com maior contaminação, encontrando escolhas alimentares mais saudáveis. Através de uma revisão sistemática da literatura parece existir uma relação entre o consumo de pescado e os níveis de metilmercúrio no organismo, que se associam a défices cognitivos em crianças cujas mães estiveram excessivamente expostas a este elemento. Porém, o peixe é também uma fonte importante de diversos nutrientes essenciais, proteínas, vitaminas, minerais e ácidos gordos com efeito protetor na exposição ao mercúrio. Torna-se necessário dotar as gestantes de conhecimento sobre o impacto do mercúrio no crescimento e desenvolvimento fetal, bem como as suas principais fontes. É importante consciencializar para o consumo de peixes pequenos e de baixo nível trófico, atendendo aos seus benefícios para a saúde materna e fetal e respetivos riscos de contaminação.

\section{PALAVRAS-CHAVE}

Exposição prenatal, Metilmercúrio, Nutrientes protetores

\section{ABSTRACT}

Pregnancy is a period of great susceptibility of exposure to environmental contaminants, such as heavy metals, including methylmercury, since it can cross the placental and blood-brain barrier, impairing fetal growth and development. This review is intended to understand mercury metabolism and its impact on the health of pregnant women and fetal development and identify foods with a higher risk of methylmercury contamination and propose healthier options. Through literature review, studies demonstrate a correlation between fish consumption and the levels of methylmercury in the body and associate these levels with cognitive deficits in children whose mothers were excessively exposed to this element. However, fish is also an essential source of several important nutrients, proteins, vitamins, minerals and fatty acids with a protective effect on exposure to mercury. It is necessary to inform pregnant women about the impact of mercury on fetal growth and development and its primary sources. It is crucial to raise awareness for the consumption of small fishes and low trophic level, taking into account their benefits for maternal and fetal health and contamination risks.

KEYWORDS

Prenatal exposure, Methylmercury, Protective nutrients

\section{INTRODUÇÃO}

Os poluentes ambientais são um problema global com impacto profundo no meio ambiente e em todos os seres vivos (1). De acordo com a Organização Mundial da Saúde, o mercúrio $(\mathrm{Hg})$ é considerado um dos dez produtos químicos com maior preocupação para a saúde pública (2) devido ao potencial neurotóxico (3), à capacidade de interferir no metabolismo de citocinas inflamatórias (4) e do selénio (Se), entre outros (5-9). Este elemento encontra-se presente no ar, água, terra e solo, bem como em organismos vivos. A exposição excessiva ao Hg está associada a diversos efeitos nefastos para a saúde, afectando principalmente o sistema nervoso central e os rins (10)
O Hg encontra-se sob diferentes formas no meio ambiente e com diferentes níveis de toxicidade. Um dos compostos mais tóxicos é o metilmercúrio (MeHg), formado a partir da metilação de formas inorgânicas de Hg por microrganismos, sendo também o composto orgânico de $\mathrm{Hg}$ mais frequentemente encontrado no meio ambiente $(7,10,11)$.

Os compostos orgânicos de Hg podem acumular-se em organismos vivos, como o peixe, de forma significativa (10), em especial nas espécies predadoras $(1,11)$. Deste modo, torna-se a principal fonte de MeHg na dieta (1, 7, 10). Proteger a cadeia alimentar da contaminação por Hg é uma tarefa importante na proteção da saúde da população 
humana e um dos grandes objetivos da segurança alimentar $(1,2$, $10,12,13)$.

Uma população significativamente afetada por este metal são as mulheres grávidas, uma vez que o mesmo é capaz de atravessar a barreira placentária e hematoencefálica, prejudicando o desenvolvimento e a saúde do feto $(2,5,8,11,12,14,15)$, especialmente em indivíduos com predisposição genética $(16,17)$ como polimorfismos (12) ou marcas epigenéticas no ADN em genes como GRIN2B, NR3C1 e BDNF (18). Alguns estudos demonstram uma associação entre a exposição pré-natal ao Hg e o desenvolvimento neurológico das crianças (10, 19-21).

Apesar do risco elevado de contaminação por $\mathrm{MeHg}$, o peixe é uma fonte importante de diversos nutrientes essenciais $(8,12,13)$, nomeadamente proteína, vitaminas, minerais, oligoelementos e ácidos gordos ómega-3 (w-3) (12), cujos estudos identificam um efeito neuroprotetor no desenvolvimento das crianças, quando consumido pelas mães durante a gravidez (12, 13, 21-23).

São objetivos deste artigo rever os mecanismos e metabolismo do $\mathrm{Hg}$ e o seu impacto na saúde humana, em especial nas gestantes e no desenvolvimento fetal. Ao mesmo tempo pretende-se identificar os alimentos com maior exposição a este metal e perceber de que forma se minimizam os seus impactos.

Para a elaboração do presente artigo, a revisão bibliográfica baseou-se na pesquisa de artigos científicos das plataformas PubMed e ScienceDirect. A pesquisa foi realizada no dia 06 de abril de 2021 , recorrendo às palavras-chave: "pregnancy mercury exposure", "methylmercury toxicity", "nutrition" e "prenatal mercury exposure". Para a adequada seleção dos artigos foram delineados alguns critérios de inclusão, nomeadamente: [1] publicação nos últimos 5 anos, [2] texto totalmente disponível de acesso gratuito, [3] relevância para o tema e [4] identificação eletrónica (DOI, PMID, ISSN ou ISSBN). Após a leitura do título foram selecionados 75 artigos e posteriormente, segundo o abstract foram selecionados 46 . Procedeu-se à sua leitura integral e 20 artigos foram excluídos por não se enquadrarem no tema, e apenas 26 cumpriam na sua generalidade os critérios de inclusão. Integrou-se posteriormente 2 artigos e 1 documento com relevância para o tema.

\section{Mercúrio e Fontes de Contaminação}

O mércurio é um xenobiótico naturalmente presente em baixos níveis no ambiente, que pode ser de origem orgânica ou inorgânica $(2,7,9)$. Este metal na sua forma inorgância encontra-se na atmosfera como $\mathrm{Hg}$ elementar $\left(\mathrm{Hg}^{\circ}\right)$ ou como $\mathrm{Hg}^{2+}$, enquanto que o $\mathrm{MeHg}\left(\mathrm{CH} 3 \mathrm{Hg}^{+}\right)$, metilado pela combinação do $\mathrm{Hg}$ com o carbono, compreende a forma orgânica mais prevalente $(2,7,9)$. Este último assume a forma mais tóxica de $\mathrm{Hg}$ e um dos químicos mais comuns no ambiente $(7,14)$. A sua principal via de absorção é a oral, distribuindo-se e acumulando-se nos organismos vivos (10).

O Hg pode ser encontrado na água, solo, ar e seres vivos e provém de fontes naturais ou humanas (10), sendo que os maiores níveis de contaminação tem origem antropogénica. A sua natureza volátil facilita a propagação pelos diversos ecossistemas (2).

O meio aquático sofre o maior impacto do $\mathrm{Hg}$ devido à deposição atmosférica, que por metilação bacteriana é convertido em MeHg, tendo assim capacidade de se acumular na cadeia alimentar aquática $(2,11,13)$. A sua concentração é mais elevada nas espécies do topo da cadeia alimentar devido à bioacumulação e biomagnificação $(7$, 11). Os níveis totais de $\mathrm{Hg}$ variam de acordo com o tamanho, espécie e origem (1). A acumulação deste metal no músculo dos peixes e crustáceos constitui um problema para o ser humano, uma vez que aumenta a sua exposição ao $\mathrm{MeHg}$ (2).
De acordo com a Comissão Reguladora (EC) da União Europeia n¹881/2006, que estabelece os níveis máximos para certos contaminantes em géneros alimentares, o limite máximo de $\mathrm{Hg}$ em peixe é de 0,5 mg/kg e $1 \mathrm{mg} / \mathrm{kg}$ para peixes predadores (24). Em Portugal e Espanha, segundo um estudo de Cabañero et al. (2005), os niveis de $\mathrm{Hg}$ na cavala, polvo, peixe-espada, sardinha e atum, encontraram-se abaixo dos máximos permitidos. No entanto, mais de $93 \%$ do Hg encontrado deve-se à forma orgânica deste metal (1). Segundo o Grupo de Estudos para a Prevenção da Exposição ao Me$\mathrm{Hg}$ (GEPREM-Hg) em Espanha, as mulheres grávidas, em lactação e as crianças até aos 14 anos devem evitar o consumo dos seguintes peixes: Atum patudo (Thunnus obesus) procedente do atlântico; Atum vermelho (Thunnus thynnus); cação (Galeorhinus galeus); Linguado (Hippoglossus hippoglossus); Lúcio (Esox lucius); marlim (Makaira species); peixe-boi (Isurus oxyrinchus); espadarte (Xiphias gladius); pata roxa (Scyliorhinus canicula); olho-de-vidro (Hoplostethus mediterraneus); tubarão (Carcharhinus species); e tintureira (Prionace glauca). Também Kimáková et al. (10) consideram o consumo de tubarão, peixe-espada e cavala proveniente do Golfo do México não apropriado para crianças, mulheres em idade fértil, mulheres grávidas e mães que amamentam.

\section{Exposição Pré-natal e Impacto no Desenvolvimento Fetal}

A alimentação materna no período prenatal é de elevada importância para o desenvolvimento fetal. A ingestão inadequada pode acarretar prejuízos não só durante o período intrauterino, mas também ao longo da vida do recém-nascido.

Baldewsingh et al. realizou um estudo prospetivo de coorte, no Suriname, no qual concluiu que 93\% das grávidas apresentavam níveis capilares significativamente elevados de Hg. No entanto, não encontraram relação com problemas no nascimento, enquanto as mulheres que tinham níveis mais baixos de $\mathrm{Hg}$ tiveram bebés com baixo peso. Esta ausência de relação pode-se dever ao efeito protetor do peixe devido à sua riqueza em Se e ácidos gordos ómega-3 (13). Patel e colaboradores desenvolveram um estudo, no Cincinnati (Ohio), com o objetivo de avaliar a associação entre os baixos níveis de $\mathrm{Hg}$ durante o desenvolvimento fetal e problemas de comportamento e défice de habilidades intelectuais em crianças. Ainda que os autores afirmem não haver evidências consistentes para efeitos adversos de uma baixa exposição ao $\mathrm{MeHg}$ durante o período pré-natal, apresentaram algumas evidências de uma associação entre uma baixa exposição ao mercúrio durante a gravidez precoce e ansiedade relatada pelos pais em crianças aos 8 anos de idade (3).

Ursinyova et al. estudaram um grupo de mães e filhos, na Eslováquia, que foram acompanhados até às 6 semanas para avaliar a exposição ao $\mathrm{Hg}$. Os resultados sugerem que a concentração de MeHg aumenta no sangue do cordão umbilical com o consumo de peixes marinhos, mesmo na população com baixo consumo de pescado, no entanto, a associação foi fraca (23). Nesse sentido, Naess e colaboradores, analisaram as concentrações de mercúrio total $(\mathrm{THg})$ no cabelo de grávidas norueguesas que consumiam bacalhau atlântico (Gadus morhua) e verificaram que o aumento do consumo daquele peixe levou a um aumento ligeiro na concentração de THg nas gestantes (25).

O estudo de coorte Tohoku Study of Child Development desenvolvido em grávidas, por Iwai-Shimada e colegas, no Japão, analisou os níveis de exposição aos diferentes elementos tóxicos, inclusive MeHg e THg, no sangue materno, cordão umbilical e placenta. Concluíram que existe associação entre as concentrações de MeHg e THg no cordão umbilical e sangue materno, o que comprova a exposição fetal. Determinaram ainda que os níveis encontrados no sangue do 
cordão umbilical eram duas vezes mais elevados que os níveis no sangue materno. Portanto, o MeHg pode ser transportado ativamente pela placenta por meio da conjugação de cisteína através do sistema transportador de aminoácidos neutros (26).

Esta maior sensibilidade dos fetos e recém-nascidos a baixos teores de MeHg, poder-se-á justificar pela menor eficácia da barreira hematoencefálica, uma vez que não se encontra totalmente formada antes dos 6 meses de idade (14), aliado a uma maior taxa de absorção gastrointestinal, excreção renal menos eficaz e ao baixo peso corporal face ao elevado consumo de alimentos por quilograma de peso corporal (9). A vulnerabilidade e imaturidade cerebral deve-se ao seu processo complexo de desenvolvimento (14). A capacidade do MeHg atravessar a placenta e a barreira hematoencefálica leva a que este chegue à parênquima cerebral e exerça um efeito deletério nas células neuronais (2) devido à acumulação nos astrócitos (14), o que permite a entrada de químicos no sistema nervoso central do bebé, que pode levar à sua acumulação cerebral e, consequentemente, aumentar o risco de atraso de desenvolvimento neurológico $(11,12,14)$.

Este processo ocorre na gravidez, contudo estende-se até ao período de amamentação, uma vez que o MeHg é excretado pelo leite materno e 95\% deste é absorvido pelo trato gastrointestinal $(2,11,14)$ e através da circulação sanguínea pode chegar a diferentes órgãos (7).

Há também evidências de que fetos de mães com doença crónica renal (DCR) são mais susceptíveis de acumular MeHg do que fetos de mães saudáveis, isso porque a captação de Hg nos rins fetais é aumentada (27).

Ulloa et al. examinaram a associação entre o MeHg e a metilação do ADN num estudo de desenvolvimento infantil das Seychelles (SCDS) (18). Foram analisados os genes BDNF, GRIN2B e NR3C1 devido ao seu papel crucial no desenvolvimento e função neuronal, já anterioriormente associados à exposição ao MeHg. Foram encontradas correlações positivas entre o Hg presente no cabelo das mães e níveis de metilação do ADN. As alterações no recetor glucocorticoide NR3C1, nomeadamente quanto à sua reduzida expressão génica, parecem estar associadas a resultados adversos no desenvolvimento neuronal. Para além dos efeitos neurotóxicos, a exposição a níveis elevados de $\mathrm{MeHg}$ pode também contribuir para a síndrome metabólica em crianças. Stratakis et al. estabeleceram essa relação no seu estudo e, com auxílio de biomarcadores de inflamação em crianças, verificaram que houve um aumento da inflamação e uma deterioração do perfil metabólico no público alvo (4).

\section{ANÁLISE CRÍTICA}

A discussão sobre os riscos e benefícios do consumo de peixe, tendo em conta a sua possível contaminação por $\mathrm{MeHg}$, torna este tema desafiante, principalmente no que se refere a estabelecer recomendações, consistentes com observações epidemiológicas, sobre o seu consumo durante a gravidez (28). Neste sentido, torna-se necessário investigar os benefícios versus os efeitos adversos do baixo consumo materno de peixe no desenvolvimento infantil (23). O consumo de pescado apresenta uma íntima ligação com o desenvolvimento neurocognitivo da criança $(19,25)$. A eliminação ou redução significativa do seu consumo pode incorrer em prejuízos no desenvolvimento neuronal do feto, uma vez que este grupo alimentar é rico em proteína (aminoácidos essenciais), vitaminas e minerais, para além de ser considerado a principal fonte dietética de ácidos gordos polinsaturados de cadeia longa $w-3$, que apresentam um potencial anti-inflamatório, benefícios cardiometabólicos e caracteristicas neuroprotetoras $(4,7,13$, 28). O ácido docosa-hexaenóico (DHA) é particularmente importante no terceiro trimeste de gravidez, período em que ocorre a sinaptogénese e o desenvolvimento dos fotorecetores no feto (22).

Sabe-se hoje que o stress oxidativo é uma das consequências de níveis elevados de MeHg pela sua capacidade de gerar espécies reativas de oxigénio, com efeitos deletérios no organismo e consequente depleção da atividade enzimática antioxidante (7). Por forma a minimizar o stress oxidativo podemos contar com um sistema exógeno de defesa, que depende de vitaminas e minerais, onde o Se se torna essencial, contribuindo para o bom funcionamento das enzimas antioxidantes e neutralização dos efeitos do MeHg. Este mineral encontra-se presente principalmente em peixes oceânicos, como a sardinha da costa portuguesa e espanhola, e em outros alimentos como a castanha do brasil $(1,5,8)$.

Para além do Se, de acordo com estudos realizados em ratos, as vitaminas $C$ e $E$ também parecem atuar neste processo através da redução do dano oxidativo, da peroxidação lipídica e no aumento da imunidade (7). Outros compostos como o Zinco (Zn) e a N-acetilcisteína (NAC) parecem atuar como fatores de proteção. O Zn é capaz de se ligar ao MeHg, diminuindo os seus efeitos tóxicos, à semelhança da NAC que funciona como um agente quelante do $\mathrm{MeHg}$. O tratamento com pólen de abelha em animais também parece promissor pela redução efetiva dos efeitos neurotóxicos do $\mathrm{MeHg}$, devido ao efeito protetor, ao nível dos neurotransmissores, bem como redução da inflamação, apoptose e excitotoxicidade do glutamato (13).

Os estudos realizados em animais podem ser um ponto de partida para a investigação destes compostos em humanos, com o intuito de atenuar os efeitos tóxicos do MeHg durante a gravidez e lactação, uma vez que não é possível erradicar o mesmo da alimentação (27). A investigação aliada a uma sensibilização e consciencialização das mulheres grávidas e em idade fértil assumem-se como estratégias em saúde na prevenção da doença.

Por forma a estabelecer um equilíbrio acerca das informações sobre o risco/benefício do peixe para a saúde, o documento Alimentação e Nutrição na Gravidez publicado este ano pela Direção Geral da Saúde vem orientar as grávidas para o consumo de pescado (29). Cabe ao nutricionista ter a capacidade de consciencializar mulheres em idade fértil, grávidas e lactantes para as recomendações de saúde pública sobre o consumo de peixe, enaltecendo a sua importância no desenvolvimento ao longo de toda a gravidez e nos primeiros anos de vida.

\section{CONCLUSÕES}

A procura pelo equilíbrio entre o consumo de peixe e a possível exposição ao metilmercúrio tem sido alvo de discussão e um grande desafio para a investigação. Torna-se necessário consciencializar as mulheres sobre o impacto do $\mathrm{MeHg}$ no crescimento e desenvolvimento fetal, as principais fontes de contaminação e recomendar o consumo de peixes de pequeno porte e de baixo nível trófico, atendendo aos seus benefícios para a saúde materna e fetal e o baixo risco de contaminação. Aliada à prevenção, é de aludir a importância de criar estratégias que minimizem o impacto do $\mathrm{MeHg}$ na saúde materna e fetal, através da promoção de um consumo seletivo de pescado, mas também na ingestão de alimentos com efeitos protetores, como é o caso do Se, o ómega-3, entre outros. O estudo de nutrientes e compostos bioativos capazes de neutralizar o stress oxidativo provocado pelo MeHg, dificultando ainda a sua passagem pela barreira placentária e hematoencefálica, é uma possível abordagem de futuro, juntamente com estudos longitudinais que permitam avaliar a longo prazo o seu impacto e assim encontrar formas de minimizar o impacto do MeHg no desenvolvimento fetal. 


\section{REFERÊNCIAS BIBLIOGRÁFICAS}

1. Cabañero Al, Carvalho C, Madrid Y, Batoréu C, Cámara C. Quantification and speciation of mercury and selenium in fish samples of high consumption in Spain and Portugal. Biol Trace Elem Res. 2005;103(1):17-35.

2. Novo JP, Martins B, Raposo RS, Pereira FC, Oriá RB, Malva JO, et al. Cellular and molecular mechanisms mediating methylmercury neurotoxicity and neuroinflammation. Int J Mol Sci. 2021;22(6):1-25.

3. Patel NB, Xu Y, McCandless LC, Chen A, Yolton K, Braun J, et al. Very low-level prenatal mercury exposure and behaviors in children: The HOME Study. Environ Heal A Glob Access Sci Source. 2019;18(1):1-12.

4. Stratakis N, Conti D V., Borras E, Sabido E, Roumeliotaki T, Papadopoulou E, et al. Association of Fish Consumption and Mercury Exposure During Pregnancy With Metabolic Health and Inflammatory Biomarkers in Children. JAMA Netw open. 2020;3(3):e201007.

5. Ralston NVC, Kaneko JJ, Raymond LJ. Selenium health benefit values provide a reliable index of seafood benefits vs. risks. J Trace Elem Med Biol. 2019;55(January):50-7. 6. Llorente Ballesteros MT, García Barrado B, Navarro Serrano I, Izquierdo Álvarez S, del Pueyo García Anaya M, González Muñoz MJ. Evaluation of blood mercury and serum selenium levels in the pregnant population of the Community of Madrid, Spain. J Trace Elem Med Biol [Internet]. 2020;57(September 2019):60-7. Available from: https://doi.org/10.1016/j.jtemb.2019.09.008.

7. Moniruzzaman M, Lee S, Park Y, Min T, Bai SC. Evaluation of dietary selenium, vitamin $\mathrm{C}$ and $\mathrm{E}$ as the multi-antioxidants on the methylmercury intoxicated mice based on mercury bioaccumulation, antioxidant enzyme activity, lipid peroxidation and mitochondrial oxidative stress. Chemosphere [Internet]. 2021;273:129673. Available from: https://doi.org/10.1016/j.chemosphere.2021.129673.

8. Castriotta L, Rosolen V, Biggeri A, Ronfani L, Catelan D, Mariuz M, et al. The role of mercury, selenium and the $\mathrm{Se}-\mathrm{Hg}$ antagonism on cognitive neurodevelopment: A 40-month follow-up of the Italian mother-child PHIME cohort. Int J Hyg Environ Health [Internet]. 2020;230:113604. Available from: https://doi.org/10.1016/j. ijheh.2020.113604.

9. Bjørklund G, Chirumbolo S, Dadar M, Pivina L, Lindh U, Butnariu M, et al. Mercury exposure and its effects on fertility and pregnancy outcome. Basic Clin Pharmacol Toxicol. 2019;125(4):317-27.

10. Kimáková T, Kuzmová L, Nevolná Z, Bencko V. Fish and fish products as risk factors of mercurexposure. Ann Agric Environ Med. 2018;25(3):488-93.

11. So SCA, Tsoi MF, Cheung AJ, Cheung T, Cheung BMY. Blood and Urine Inorganic and Organic Mercury Levels in the United States from 1999 to 2016. Am J Med [Internet]. 2021;134(1):e20-30. Available from: https://doi.org/10.1016/j.amjmed.2020.06.023.

12. Brumatti LV, Rosolen V, Mariuz M, Piscianz E, Valencic E, Bin M, et al. Impact of methylmercury and other heavy metals exposure on neurocognitive function in children aged 7 years: Study protocol of the follow-up. J Epidemiol. 2021;31(2):157-63. 13. Baldewsingh GK, Wickliffe JK, van Eer ED, Shankar A, Hindori-Mohangoo AD, Harville EW, et al. Prenatal mercury exposure in pregnant women from suriname's interior and its effects on birth outcomes. Int J Environ Res Public Health. 2020;17(11):1-13. 14. Ben Bacha A, Norah AO, Al-Osaimi M, Harrath AH, Mansour L, El-Ansary A. The therapeutic and protective effects of bee pollen against prenatal methylmercury induced neurotoxicity in rat pups. Metab Brain Dis. 2020;35(1):215-24.

15. Cambier S, Fujimura M, Bourdineaud JP. A likely placental barrier against methylmercury in pregnant rats exposed to fish-containing diets. Food Chem Toxicol [Internet] 2018;122(September):11-20. Available from: https://doi.org/10.1016/j.fct.2018.09.066. 16. Karin Wahlberg, Love TM, Pineda D, Engström K, Watson GE, Thurston SW, et al. Maternal polymorphisms in glutathione-related genes are associated with maternal mercury concentrations and early child neurodevelopment in a population with a fish-rich diet. Environ Int. 2018;115:142-9.

17. Julvez J, Davey Smith G, Ring S, Grandjean P. A Birth Cohort Study on the Genetic Modification of the Association of Prenatal Methylmercury With Child Cognitive Development. Am J Epidemiol. 2019;188(10):1784-93.

18. Cediel Ulloa A, Gliga A, Love TM, Pineda D, Mruzek DW, Watson GE, et al. Pre- natal methylmercury exposure and DNA methylation in seven-year-old children in the Seychelles Child Development Study. Environ Int [Internet]. 2021;147(December 2020):106321. Available from: https://doi.org/10.1016/j.envint.2020.106321.

19. Hibbeln CJR, Spiller P, Brenna JT, Golding J, Holub BJ, Harris WS, et al. Relationships between seafood consumption during pregnancy and childhood and neurocognitive development: Two systematic reviews. Prostaglandins Leukot Essent Fat Acids [Internet]. 2019;151(October):14-36. Available from: https://doi.org/10.1016/j. plefa.2019.10.002

20. Raposo R da S, Pinto DV, Moreira R, Dias RP, Fontes Ribeiro CA, Oriá RB, et al. Methylmercury Impact on Adult Neurogenesis: Is the Worst Yet to Come From Recent Brazilian Environmental Disasters? Front Aging Neurosci. 2020;12(November):1-7.

21. Ripley S, Robinson E, Johnson-Down L, Andermann A, Ayotte P, Lucas M, et al. Blood and hair mercury concentrations among Cree First Nations of Eeyou Istchee (Quebec, Canada): time trends, prenatal exposure and links to local fish consumption. Int J Circumpolar Health [Internet]. 2018;77(1):1-9. Available from: https://doi.org/10 .1080/22423982.2018.1474706.

22. Nunes E, Cavaco A, Carvalho C. Exposure assessment of pregnant portuguese women to methylmercury through the ingestion of fish: Cross-sectional survey and biomarker validation. J Toxicol Environ Heal - Part A Curr Issues. 2014;77(1-3):133-42. 23. Ursinyova M, Masanova V, Unnakova I, Murinova LP, Patayova H, Rausova K, et al. Prenatal and Early Postnatal Exposure to Total Mercury and Methylmercury from Low Maternal Fish Consumption. Biol Trace Elem Res. 2019;191(1):16-26.

24. Reg. no1881/2006. Regulamento (CE) no 1881/2006 da Comissão de 19 de Dezembro de 2006 que fixa os valores máximos de certos contaminantes presentes em géneros alimentícios. Jornal Oficial da União Europeia. 2006. p. L 364/5-L 364/24. 25. Næss S, Kjellevold M, Dahl L, Nerhus I, Midtbø LK, Bank MS, et al. Effects of seafood consumption on mercury exposure in Norwegian pregnant women: A randomized controlled trial. Environ Int [Internet]. 2020;141(January):105759. Available from: https:// doi.org/10.1016/j.envint.2020.105759.

26. Iwai-Shimada M, Kameo S, Nakai K, Yaginuma-Sakurai K, Tatsuta N, Kurokawa $\mathrm{N}$, et al. Exposure profile of mercury, lead, cadmium, arsenic, antimony, copper, selenium and zinc in maternal blood, cord blood and placenta: The Tohoku Study of Child Development in Japan. Environ Health Prev Med. 2019;24(1):1-11.

27. Moss RF, George HS, Nijhara S, Orr SE, Joshee L, Barkin JL, et al. Chronic kidney disease in pregnant mothers affects maternal and fetal disposition of mercury. Reprod Toxicol [Internet]. 2020 Apr;93(1):137-45. Available from: https://linkinghub.elsevier. com/retrieve/pii/S0890623820300186.

28. Groth E. Scientific foundations of fish-consumption advice for pregnant women: Epidemiological evidence, benefit-risk modeling, and an integrated approach. Environ Res. 2017;152:386-406.

29. Teixeira D, Marinho R, Inês Mota, Castela I, Morais J, Pestana D, et al. Alimentação e Nutrição na Gravidez 2021 [Internet]. Lisboa: Direção-Geral da Saúde; 2021. p. 80. Available from: www.dgs.pt. 